\title{
SPECT myocardial perfusion imaging as an endpoint
}

\author{
Melody Sherwood, MD, ${ }^{a}$ Fadi G. Hage, MD, FACC, ${ }^{b}$ Jack Heo, MD, FACC, ${ }^{a}$ \\ Leslee J. Shaw, PhD, FACC, FASNC, ${ }^{c}$ Manuel D. Cerqueira, MD, FACC, FASNC, ${ }^{d}$ \\ and Ami E. Iskandrian, MD, MACC, FASNC ${ }^{\mathrm{a}}$
}

The wide acceptance of SPECT myocardial perfusion imaging (MPI) in patient care has led increasingly to its use as an endpoint in single- or multi-center studies. ${ }^{1-6}$ These studies differ in scope and complexity and may involve issues beyond reproducibility and repeatability, not to say that these are not important. We shall look at two examples to bring up salient features. ${ }^{3,4}$

Two NIH-NHLBI sponsored studies "the international study of comparative health effectiveness with medical and invasive approaches (ISCHEMIA)" trial will be conducted in 8,000 patients from $\sim 400$ worldwide centers and "The effect of transendocardial delivery of autologous bone marrow mononuclear cells in chronic heart failure (The FOCUS-CCTRN Trial)"' in 92 patients was completed in five centers are examples worth considering because of marked variability in design and requirements. ${ }^{3,4}$

The ISCHEMIA trial will compare a strategy of catheterization-guided care to no catheterization-guided care. Importantly, only patients with moderate-severe ischemia will be enrolled, defined with stress MPI as $\geq 10 \%$ ischemic myocardium. All patients will undergo a blinded coronary computed tomographic angiography to exclude significant left main stenosis and to rule out non-obstructive $\mathrm{CAD}^{3}$

The FOCUS Trial examined whether administration of bone marrow mononuclear cells, through transendocardial injections, would improve myocardial perfusion, reduce left ventricular (LV) end-systolic volume, and enhance maximal oxygen consumption in patients with

From the University of Alabama at Birmingham, ${ }^{\text {a }}$ Birmingham Veterans Affairs Medical Center, ${ }^{\mathrm{b}}$ Birmingham, AL; Emory University School of Medicine, ${ }^{c}$ Atlanta, GA; and Cleveland Clinic Foundation, ${ }^{\mathrm{d}}$ Cleveland, OH.

Reprint requests: Ami E. Iskandrian, MD, MACC, FASNC, University of Alabama at Birmingham, 318 LHRB, Birmingham, AL 35294; aiskand@uab.edu.

J Nucl Cardiol 2012;19:891-4.

$1071-3581 / \$ 34.00$

Copyright (c) 2012 American Society of Nuclear Cardiology.

doi:10.1007/s12350-012-9583-0 coronary artery disease (CAD) or LV dysfunction, and limiting heart failure or angina. ${ }^{4}$

In the ISCHEMIA trial, the inclusion criteria required the presence of ischemia involving at least $10 \%$ of LV myocardium as this trial follows prior stable ischemic heart disease trials by focusing on higher risk CAD patients with a substantial ischemic burden, but importantly is based on the current equipoise as to whether the degree of ischemia remains vital to therapeutic effectiveness; notably a strategy that includes revascularization, while in the FOCUS trial, one of the three primary endpoints required at least $10 \%$ absolute reduction in ischemic burden. Unfortunately, requiring $10 \%$ ischemic burden excluded too many patients and the final third of enrolled patients included those with both fixed and reversible defects. Although an echocardiographic core lab was used to standardize the echo measured variables, SPECT studies were interpreted only at the site of enrollment. On-site reading tends to overcall ischemia when compared to core lab reading. Of note the ISCHEMIA trial required one study at entry while the FOCUS trial required two studies, one before and the second 6 months after treatment.

How is $10 \%$ of the myocardium measured? Is the use of $10 \%$ appropriate in both the studies? While these questions might sound simple; in point of fact they are anything but simple and they will be the subject of this editorial viewpoint (Table 1). Just to repeat, in the ISCHEMIA trial, the $10 \%$ threshold for ischemia was one of the entry criteria while in the FOCUS trial it was one of the endpoints.

\section{IMAGE INTERPRETATION}

Interpretation of MPI is easier and more reproducible when the images are of high quality or if they are clearly normal regardless of whether visual or automated methods are used. ${ }^{7-16}$ Quality is difficult to define but it is affected by many steps during acquisition and processing including patient motion, filtering, collimation, alignment, and scaling, issues that are not corrected by attenuation correction algorithms whether done by CT or external transmission source. It is for this reason 
Table 1. Factors to be considered in using MPI as endpoint

\author{
Single versus serial testing \\ Method of interpretation \\ Visual versus automated \\ Scoring system versus polar maps \\ Extent versus severity \\ Relative versus absolute change \\ Inclusion criteria \\ Type of stress test
}

that in multi-center trials, a core laboratory is recommended to assure uniform processing. Even when everything is performed consistent with current guideline statements, there remain unpredictable factors of attenuation artifacts and tracer activity in the liver and more importantly in an adjacent loop of the bowel. These factors could vary in the rest or stress studies and that is one of the reasons of why studies that include serial testing are inherently more difficult than studies based on a single study. ${ }^{7,8}$ In patient care studies, serial images should be reviewed side-by-side so that the reader can adjudicate the contributions of the extraneous factors on image interpretation (whether done by visual or automated analysis, see below). This crucial step of side-by-side reading is often not used or allowed in research studies, which in our opinion is unfortunate as this direct simultaneous comparison with treatment blinding provides the best opportunity to assess the factors listed above and how they may impact on serial study measurements.

How should the images be interpreted? There are several ways but the common approaches include the use of a segmental scoring system or quantitative polar maps. It should be clear that even the automated methods require some reader supervision and blinded acceptance of computer generated scores is ill-advised. Automated or more precisely supervised automated methods have better performance in terms of reproducibility (same image processed twice) and repeatability (two sequential acquisitions after tracer injection) than visual analysis, which in research studies translate to a smaller sample size. ${ }^{7,11}$

\section{SCORES AND MAPS}

The scoring system could be generated by visual or automated methods. The summed stress score (SSS), summed rest score (SRS), and summed difference score (SDS) are global scores that reflect total, fixed, and reversible abnormality, respectively, and could readily be converted to \% LV myocardial abnormality [if a normal SSS is 68 for example (17-segment model where a score of 4 in a segment indicates a normal tracer activity; $17 \times 4=68$ ), then SSS of 34 means that the perfusion abnormality involves $50 \%$ of LV myocardium]. ${ }^{7,8}$

The SSS or \% abnormality is a global number and thus does not specify the vascular territory or whether the abnormality is in 1,2 , or 3 vascular territories (although such could be done). Though the SSS (or SDS or SRS) could be converted to \% LV abnormality, this may or may not be similar to the \% abnormality obtained by polar maps because a given SSS may represent a mild abnormality involving a number of segments or a severe abnormality involving much smaller number of segments in which case the polar maps will show a larger abnormality in the first than the second patient.

The polar maps (or bull's eye images), which was used in the COURAGE (optimal medical therapy with or without percutaneous coronary intervention to reduce ischemic burden: results from the COURAGE trial) and BARI2D (a randomized trial of therapies for type 2 diabetes and coronary artery disease) nuclear sub-studies have provided information on $\%$ total abnormality (fixed + reversible), \% reversible abnormality (ischemia) and \% fixed abnormality (scar). A combination of extent and severity known as total perfusion deficit (TPD, used in the COURAGE trial), or separate extent and severity maps could be generated using polar map displays which may provide improved delineation due to its ability to quantify defect size relying on computer pixel as the unit of analysis. It also remains likely that therapeutic intervention may elicit greater changes to defect severity versus extent. It is conceivable that some interventions might decrease the severity but not the extent of ischemia but as most ischemic abnormalities are in the mild-moderate range, changes in severity alone are likely to be difficult to assess or at least to demonstrate. The results from of the polar maps like the scores represent global abnormality though the 2D and $3 \mathrm{D}$ images nicely display the location in relation to vascular territories and hence such results could be obtained per each vascular territory if desired.

There are several commercial softwares that can be used for automated analysis. ${ }^{10-15}$ Although, generally, there is agreement between them in assessing defect size and reversibility, caution should be used in not using them inter-changeably or extrapolating threshold obtained by one method to define small or large abnormality into other softwares. For example $10 \%$ abnormality might be considered large by one software but small-moderate by another. In general, the packages which rely on extent have higher \% myocardial involvement than those that incorporate severity. 


\section{GLOBAL VERSUS REGIONAL ASSESSMENT}

The traditional way of comparing two sets of images (such as pre- and post-intervention) is to measure the abnormality from each set of images separately (blinded to clinical information except gender) although $3 \mathrm{D}$ registration of the two sets of the images is more appealing and feasible especially to detect subtle changes. Even, when such a detailed analysis is attempted, one needs to keep in mind that a lack of a change in global score is no assurance that a change did not occur such as improvement in one area coupled with worsening in another area such that the net effect cancels each other. This is especially true when serial studies are done with interventions that involve targeted therapy such as coronary revascularization by any means. In such cases, changes in different locations (such as septum, anterior wall, inferior wall, lateral wall, and apex) might be very meaningful.

It is well known that some fixed perfusion defects, especially if mild, when using Tc-labeled tracers (which lack the ability to redistribute over time unlike thallium201) might be due to severe reduction in resting myocardial blood flow (MBF) and hence might improve following interventions that are known to increase MBF such as coronary revascularization, angiogenesis, stem cell therapy, or even pharmacological treatment. Therefore, assessment of resting perfusion is not only important as an endpoint but also in patient selection; i.e., patients with mild fixed defects should not be excluded from studies that evaluate effect of interventions that are presumed to improve MBF. One should be careful not to use "ischemia" and "viable myocardium" inter-changeably as viable myocardium could exist with no ischemia by stress MPI. ${ }^{1,2}$

\section{PATIENT SELECTION}

The inclusion criteria, which determine patient recruitment and for that matter the length and costs of the study are major issues and at times have no easy solutions. Ideally, the patients should be representative of patients with a given problem but that is easier said than done. For example, patients with classic or typical symptoms are likely to be triaged to coronary angiography and interventions. The same is also true for patients with a large ischemic burden who have good target vessels bases on accumulated evidence from the past three decades. These two reasons alone would suggest that patients included in trials will have no or mild symptoms or small ischemia (in COURAGE, nearly two-thirds of patients had mild ischemia). It is more difficult to discern a change on serial testing when the initial abnormality is small. From a pragmatic viewpoint, one can argue that it does not really matter if the intervention does not improve the perfusion in such patients because they are already at low risk and have no or minimal symptoms. (Obviously, there is a repeatability factor where if someone has $5 \%$ ischemia, then any change may be within the repeatability factor.)

Equally as important is the incomplete inclusion of imaging endpoints in many of the published trials. When only a subset of patients are imaged, it is crucial to critically analyze differences between the groups as there is usually a selection bias resulting in different populations between the entire cohort and those imaged. Importantly, when imaging results fail to define a difference in therapeutic intervention, there are issues with statistical power that limit the interpretation of ischemic findings, and many times it is based on technology availability at the centers, which may select tertiary high volume centers including more patients with the imaging endpoint. The other inclusion/exclusion criteria are based on LVEF and prior myocardial infarction. The reason being that detection of ischemia is more difficult when there is significant scar. This assumption is, however, not straight forward because the depressed EF could be due to valvular or myopathic processes putting these patients at higher risk even with a small amount of ischemia.

What should be the expectation of improvement in perfusion when this variable is used as an endpoint? Some studies used a value based on 95\% CI around the mean change in serial testing from independent group of patients. Others required that the change to be greater than in a placebo group when such a group was also studied. The improvement is either expressed as \% relative change or $\%$ absolute change. For example, a reduction from $10 \%$ to $5 \%$ after an intervention represents a 50\% relative improvement or 5\% absolute improvement. The relative change is appealing as it is not dependent on baseline absolute value. Further, a $10 \%$ reduction from a large baseline value (for example, $60 \%$ of the myocardium) may still mean that there is a large residual abnormality after treatment and hence it is unlikely that the risk will be any different! Ideally, a reduction from a large ischemic abnormality to no or very small abnormality (together with symptomatic improvement) is the most desirable endpoint (though seldom achieved) because many studies show that patients with no or small abnormality are at low risk for future cardiac events. Such a dramatic change could be seen, however, after complete revascularization either by coronary artery bypass grafting or percutaneous coronary interventions. ${ }^{1,2}$ One should also be careful to distinguish a statistically significant change from a clinically relevant change, but that is obviously not unique to MPI as it is true in most interventional studies. 


\section{TYPE OF STRESS TEST}

The type of stress test is another issue that does not receive adequate attention. Often symptomatic patients with other co-morbidities undergo vasodilator stress MPI. The concern is that even if these patients could exercise, their exercise level and performance could be different before and after an intervention that presumably improves MBF and relieves angina such as the FOCUS trial. Obviously, there are mechanisms that could be used in such circumstances but here the issue is whether the stress modality is one that is likely to show a protective effect of collaterals or for that matter new channels by stem cells therapy or does it promote coronary steal and thus likely to show unintended effect of these channels? We know that collateralized occluded vessels might show a large reversible abnormality during vasodilator MPI, with even ST-segment shifts. ${ }^{1,2}$ This issue deserves to be studied as it is crucial as to which stress test to use.

\section{CONCLUSIONS}

The ISCHEMIA trial requires one stress MPI that shows a large ischemia by software that in prior studies has been shown to predict a poor outcome. The FOCUS trial involved two sets of images and thus required more complex analysis. We hope attention to these details will help in future trials including those with stem cell therapy to be certain that MPI provides a meaningful endpoint based upon global and regional assessments. In the future, protocols that require two sets of images or more need also to consider of how best to keep the radiation risk as low as possible.

\section{References}

1. Iskandrian $\mathrm{AE}, \mathrm{Garcia} \mathrm{EV}$, editors. Atlas of nuclear cardiology: Imaging companion to Braunwald's heart disease. Philadelphia, PA: Elsevier; 2012.

2. Iskandrian AE, Garcia EV, editors. Nuclear cardiac imaging: Principles and applications. 4th ed. Oxford: Oxford University Press; 2008
3. https://www.ischemiatrial.org. Accessed 31 May 2012

4. Perin EC, Willerson JT, Pepine CJ, et al. Effect of transendocardial delivery of autologous bone marrow mononuclear cells on functional capacity, left ventricular function, and perfusion in chronic heart failure. The FOCUS-CCTRN Trial. JAMA 2012; 317:1702-6.

5. Shaw LJ, Cerqueira MD, Brooks MM, Althouse AD, et al. Impact of left ventricular function and the extent of ischemia and scar by stress myocardial perfusion imaging on prognosis and therapeutic risk reduction in diabetic patients with coronary artery disease: Results from the bypass angioplasty revascularization investigation 2 diabetes (BARI 2D) trial. J Nuc Cardiol 2012. doi:10.1007/ s12350-012-9548-3

6. Shaw LJ, Berman DS, Maron DJ, et al. Optimal medical therapy with or without percutaneous coronary intervention to reduce ischemic burden: Results from the clinical outcomes utilizing revascularization and aggressive drug evaluation (COURAGE) trial nuclear substudy. Circulation 2008;117:1283-91.

7. Slomka P, Xu Y, Berman DS, Germano G. Quantitative analysis of perfusion studies: Strengths and pitfalls. J Nucl Cardiol 2012; 19:338-46.

8. Germano G, Kavanagh PB, Slomka PJ, Berman DS. Tracking a therapeutic response: How reliable are serial measurements of LV perfusion and function? J Nucl Cardiol 2012;19:360-3.

9. Iskandrian AE, Garcia EV, Faber T. Analysis of serial images: A challenge and an opportunity. J Nucl Cardiol 2008;15:23-6.

10. Faber TL, Modersitzki J, Folks RD, et al. Detecting changes in serial myocardial perfusion SPECT: A simulation study. J Nucl Cardiol 2005;12:302-10.

11. Xu Y, Hayes S, Ali I, et al. Automatic and visual reproducibility of perfusion and function measures for myocardial perfusion SPECT. J Nucl Cardiol 2010;17:1050-7.

12. Ficaro EP, Lee BC, Kritzman JN, et al. 4DM: The Michigan method for quantitative nuclear cardiology. J Nucl Cardiol 2007; 14:455-65.

13. Germano G, Kavanagh PB, Slomka PJ, et al. Quantitation in gated perfusion SPECT imaging: The Cedars-Sinai approach. J Nucl Cardiol 2007; 14:433-54.

14. Liu YH. Quantification of nuclear cardiac images: The Yale approach. J Nucl Cardiol 2007;14:483-91.

15. Garcia EV, Faber TL, Cooke CD, et al. The increasing role of quantification in clinical nuclear cardiology: The Emory approach. J Nucl Cardiol 2007;14:420-32.

16. Mahmarian JJ, Cerqueira MD, Iskandrian AE, et al. Regadenoson induces comparable left ventricular perfusion defects as adenosine: A quantitative analysis from the ADVANCE MPI 2 trial. JACC Cardiovasc Imaging 2009;2:959-68. 